\title{
VON SONNENKÖNIGEN UND RESIDENZEN - VORDERASIATISCHE TRENDS IN DER AMARNA-REVOLUTION
}

\author{
Oskar Kaelin \\ (University of Basel, Switzerland)
}

\begin{abstract}
ABRISS
Echnatons Reformen werden meist als rein innerägyptischer Wandel betrachtet. Doch die Späte Bronzezeit war kosmopolitisch. Über Generationen gab es intensive, familiäre Beziehungen zwischen den Höfen der Grosskönige, in deren Selbstdarstellung öfters eine Hybridisierung festgestellt werden kann. Entsprechend sollten Elemente von Echnatons Revolution auch als eine Auseinandersetzung mit Trends in Vorderasien gesehen werden: der Bau einer königlichen Residenzstadt und die Schaffung des nicht-anthropomorphen Strahlenaton als neuer Gottheit.
\end{abstract}

SCHL ÜSSELWORTE

Echnaton, Amarna, Späte Bronzezeit, Ikonographie, Flügelsonne, Residenzstädte

\section{ON SUN KINGS AND RESIDENCES - NEAR EASTERN TRENDS IN THE AMARNA-REVOLUTION}

\begin{abstract}
Akhenaten's reforms are usually seen as purely internal Egyptian change. But the Late Bronze Age was cosmopolitan. For generations, there were intense family relations between the courts of the great kings, in whose self-presentation a hybridization was established. Accordingly, elements of Akhenaten's revolution should be seen as an engagement with trends from the Ancient Near East: the construction of a royal residential city and the creation of the non-anthopomorphic sun-disc with arms as a new deity.
\end{abstract}

\section{KEYWORDS}

Akhenaten, Amarna, Late Bronze Age, iconography, winged disc, residential cities

Die Amarna-Revolution, Amenophis IV./Echnatons Reformen, die in wenigen Etappen und Jahren von der traditionellen ägyptischen Religion zur Aton-Religion, vom Polytheismus über Henotheismus zum Monotheismus führte, wurde meist als innerägyptischer Wandel beschrieben. Je nach Standpunkt sah man sie als Produkt eines Genies, Fanatikers, Ketzers, Revolutionärs oder Reaktionärs. ${ }^{1}$

$\mathrm{Zu}$ Beginn seiner Regierung wurde Amenophis IV. noch ganz wie ein traditioneller ägyptischer Herrscher inszeniert, z.B. in Darstellungen vom Pharao beim Erschlagen der Feinde, beim Libieren vor seinen Eltern, bei der Darbringung von Opfern für anthropomorphe (Sonnen-)Götter oder mit einer Göttin unter einer Flügelsonne sitzend. Früh in seiner Regierung fokussierte er auf solare Gottheiten, respektierte aber zunächst ihre Vielfalt. Aton (Jtn), als Sonnenscheibe ist in Texten seit dem Alten Reich belegt. Die Vorstellung, dass der Herrscher über alles regiert was Aton umkreist, findet sich seit dem Mittleren Reich. Unter Amenophis III. erfuhr Aton eine immer stärkere Beachtung und während die Sonnenscheibe bis dahin (nur) als Symbol oder Vertreter eines Sonnengottes galt. wurde sie nun zusehends zur eigenständigen Gottheit erhoben. Die Förderung Atons scheint von Anbeginn der Regierungszeit Echnatons sein Ziel gewesen zu sein. ${ }^{2}$

\footnotetext{
${ }^{1}$ Hoffmeier 2015: 1-4. Görg 2007: 104-107. Hornung 2003: 9-27. Assmann 1984: 232-257. OEAE s.v. Akhenaten.

${ }^{2}$ Hoffmeier 2015: 76-87. Redford 1978. Redford 1980: 23-24.
} 
In Karnak entstand ein Heiligtum für Re-Harachte-Aton; Amun wurde aber noch respektiert. Jtn (Aton) die "Scheibe" bekam einen erklärenden, langen, "lehrhaften" Namen, und ab dem 3. Regierungsjahr wird wie bei einem Pharao der Name Atons von einer Kartusche umschlossen, der Gott folglich wie ein König behandelt. ${ }^{3}$ Im 4. oder 5. Regierungsjahr feierte Echnaton für Aton ein Sedfest, das üblicherweise im 30. Regierungsjahr eines Herrschers seiner Erneuerung diente. Mit diesem Sedfest wurde das Königtum des Gottes Aton (und indirekt auch die Herrschaft Echnatons) erneuert und propagiert. ${ }^{4}$ Mit dem 5. Regierungsjahr beginnen die Arbeiten zur neuen Residenzstadt Achet-Aton, wo bis zum Ende von Echnatons Regierung Aton zelebriert wird. Mit dem Umzug begann vermutlich auch die Beseitigung von Darstellungen und Schreibungen Amuns und von Pluralformen in der Schreibung von Göttern, die Meidung, sie anthropomorph darzustellen, und der Verzicht auf gewisse Elemente des Totenkultes. ${ }^{5}$ Tempel und Anlagen für Aton wurden auch an anderen Orten Ägyptens, Nubiens und des Sinai gebaut. ${ }^{6} \mathrm{Ob}$ sich jede Etappe erst aus der vorherigen ergab oder ob ein "atonistischer Fünfjahresplan" existierte, um in wenigen Jahren die neue Ideologie umzusetzen, muss offen bleiben. Auf alle Fälle sind die sich steigernden Etappen des Projektes "Aton" didaktisch bzw. propagandistisch gut gewählt.

Echnaton machte aus der real sichtbaren Sonnenscheibe Aton einen universalen, omnipräsenten Sonnengott, der den Herrschaftsanspruch und solaren Bezug Pharaos in der internationalen Welt der Späten Bronzezeit zum Ausdruck brachte. Echnaton war Atons Sohn; er identifizierte sich zwar nicht vollständig mit ihm, war aber der einzige, der Atons Absichten kannte und ihm dienen konnte. "He and the Disc were alone and supreme in the universe, over all men: there were no other gods. He was 'the living Disc - there is none other than him!" Echnaton persönlich verfasste zu Atons Ehren Hymnen, die ihn als "global player" zelebrierten, seine universale Zuständigkeit und Unabdingbarkeit für Welt und Schöpfung unterstrichen: „Du erschaffst die Erde nach deinem Wunsch, und zwar ganz allein, mit allen Menschen, mit allem Vieh und Wild, die auf der Erde und auf den Beinen laufen oder sich erheben und mit ihren Flügeln fliegen. Selbst in den Fremdländern Syrien und Nubien, die zu Ägypten gehören, setzt du jeden Mann an seinen Platz und sorgst für seinen Unterhalt ... Die Zungen sind verschieden im Sprechen, ebenso wie ihre (= der Fremdlandbewohner) Wesensart, und auch ihre Hautfarbe ist unterschiedlich, weil du so die Fremdlandbewohner unterscheidest. Du schaffst den Nil in der Tiefe und bringst von ihm hervor, so viel du willst, um die Untertanen leben zu lassen. ... Sogar alle Fremdlandbewohner, die fern sind, erhältst du am Leben, denn du hast einen Nil an den Himmel gesetzt, damit er zu ihnen herabfällt, damit er auf den Bergen Wellen schlägt wie das Meer, um ihre Felder zu bewässern, indem (er) sie berührt." Für den Weltenherrscher Aton wurde Achet-aton die Residenstadt. ${ }^{8}$

In der Amarna-Zeit, generell im Neuen Reich, gab es einen regen internationalen Austausch. Ägypten war Mitglied im "Club der Grossmächte", zu dem Babylonien, Mitanni, die Hethiter und später die Assyrer gehörten. Die Beziehungen zwischen den Höfen wurden durch Heiratspolitik, Geschenke und Diplomatie stabilisiert und intensiviert, oft indem der ägyptische König Prinzessinnen aus anderen Reichen heiratete. Das ägyptische Königshaus war über mehrere Generationen mit dem mitannischen Herrscherhaus in Nordsyrien/-irak (Amenophis II., Thutmosis IV. mit Artatama I., Amenophis III. mit Šuttarna II. und Tušratta).

\footnotetext{
${ }^{3}$ Hoffmeier 2015: 62-91 (Anfänge), 91-117 (Karnak). Hornung 2003: 41-47. LÄ s.v. Aton.

${ }^{4}$ Hoffmeier 2015: 117-125. Hornung 2003: 48-49.

${ }^{5}$ Hoffmeier 2015: 136-159. Hornung 2003: 69-88. RedFord 1980: 27-28.

${ }^{6}$ HOFFMEIER 2015: 91-107.

${ }^{7}$ REDFORD 1980: 25-26.

${ }^{8}$ BAYER 2007: 16f (Zitat). Hoffmeier 2015: 211-237. Hornung 2003: 81-98.
} 
Ebenso bestanden dynastische Verbindungen Ägyptens zum kassitischen Babylon (Thutmosis IV. mit Karaindaš, Amenophis III. mit Kurigalzu I und Kadašman-Enlil I., Echnaton mit Burnaburiaš II.), das seinerseits wiederum mit Elam liiert war. ${ }^{9}$

Echnaton wuchs in einer Zeit auf, in der gerade an den kosmopolitischen Höfen neueste Trends wahrge- und manchmal übernommen wurden. Objekte in einem "intercultural style" entstanden, die zwischen den Höfen ausgetauscht wurden und zu einer Angleichung der künstlerischen Präferenzen führten: "The exchange of daughters in interdynastic marriages literally ,hybridizes' the royal gene pool, breaking down barriers between royal families and binding them together in a manner analogous to the visual hybridity and universalizing iconography of the koine. " ${ }^{10}$ Daher sollte Echnatons "Revolution" nicht nur als isolierte, intern ägyptische, persönlich bzw. innen- und/ oder aussenpolitisch motivierte Entwicklung, sondern als Teil von und Reaktion auf kosmopolitische Trends betrachtet werden.

In dieser Späten Bronzezeit hatte das seit dem 3. Jt. v. Chr. existierende Sonnenkönigtum Ägyptens klare Konkurrenz bekommen. Es war nicht mehr das einzige Königtum mit solaren Bezügen, die Flügelsonne nicht mehr exklusives Logo des ägyptischen Herrschers. Echnaton formulierte die traditionelle Verbindung des ägyptischen Königs mit der Sonne neu, setzte die sichtbare Sonne Aton ins Zentrum und inszenierte ihn - um auf dem Stand der Zeit zu sein - mit Elementen, die sich an Trends aus dem Vorderen Orient orientierten.

\section{Der nicht-anthropomorphe Strahlenaton}

Die Verbindung zwischen (vergöttlichtem) König und Sonnengott gehörte in Ägypten seit der 1. Dynastie zu den Konstanten ägyptischer Königsideologie. ${ }^{11}$ Der Sonnengott war ein globaler, die ganze Welt überschauender Gott, was dem universalistischen Herrschaftsanspruch der ägyptischen Königsideologie entsprach. Ab der 4. Dynastie galt der König in der Königstitulatur als "Sohn des Re", was seine Göttlichkeit und seinen solaren Aspekt betonte. Während der 5. Dynastie wurden von mehreren Königen Sonnenheiligtümer gebaut, deren Namen eine Verbindung mit Re bezeugen und deren wichtigste Installation wahrscheinlich ein Obelisk war. Zur selben Zeit werden die Pyramidenbauten für die Herrscher bescheidener. ${ }^{12} \mathrm{Ab}$ der 6 . Dynastie finden sich regelmässig Darstellungen der geflügelten Sonnenscheibe, dem "Zentralsymbol der pharaonischen Monokratie". Trotz visueller Einfachheit war es von Anfang an komplex aufgeladen. "Mit ihren Flügeln symbolisiert sie das Weltumspannende der Herrschaft über 'Alles, was die Sonne umkreist', und mit der Sonnenscheibe die Idee der Einheit: Eine Sonne, ein Herrscher. Wie die Sonne die Götterwelt, so zentriert der König die Menschenwelt."13 Die geflügelte Sonnenscheibe verband Komponenten zweier Hauptgottheiten: die Sonnenscheibe für Re plus die Flügel eines Falken für Horus. Sie repräsentierte Horus-Behedeti, später Re-Harachte und andere mit dem Königtum verbundene Götter. Die Beziehung des Herrschers zum Sonnengott und die Flügelsonne als Symbol blieben bis zum Ende der pharaonischen Zeit zentrale Komponenten der ägyptischen Königsideologie. ${ }^{14}$

\footnotetext{
${ }^{9}$ Brinkman 2017: 11-13. Miller 2017. Roaf 2017: 182-195. Podany 2010: 163-242. Klinger 2006: $313-315$. BRINKMAN 1976: 240.

${ }^{10}$ Podany 2010: 243-258. Feldman 2006: 27 (Zitat und generell zum “intercultural style”). GeStoso Singer 2006.

${ }^{11}$ Hoffmeier 2015: 7-21. Gundlach 1998: 11-12, 137-142.

${ }^{12}$ Hoffmeier 2015: 24-31.

${ }^{13}$ Hoffmeier 2015: 21-24. Assmann 1993: 17 (Zitate).

${ }^{14}$ Hoffmeier 2015: 32-61. Westendorf 1986. LÄ s.v. Flügelsonne.
} 
Im Vordern Orient waren die Beziehungen zwischen Herrschaft und Sonne (sowie die Vergöttlichung der Herrscher) weniger dominant. In der Akkad-Zeit unter Naramsin findet sich die Assoziation des Herrscher mit der Sonne und seine Vergöttlichung erstmals auch in Mesopotamien. Die Vergöttlichung des mesopotamischen Königs endete mit dem Ur IIIReich. ${ }^{15}$ Hingegen findet sich die Verbindung zum Sonnengott in der altbabylonischen Zeit weiter bei mesopotamischen, syrischen und hurrischen Herrschern. Der Herrscher gehörte z.B. zur Familie des Sonnengottes, betrat in Mythen die Region des Sonnengottes, wurde von ihm geschützt, und ist auch in der Titulatur als „Sonne“ bzw. „Sonnengott" seines Landes bezeichnet worden. ${ }^{16}$

Während des ägyptischen Mittleren Reiches verbreitete sich neben anderen Motiven auch die Flügelsonne in der Levante, Nordsyrien und Kleinasien. Siegel mit Flügelsonne kennen wir z.B. von altbabylonischen Herrschern und deren Entourage aus Byblos, Yamchad, Karkemisch und Ebla. Mit der Verbreitung "popularisierte" sich das Motiv, war ein Statussymbol, das auf nicht-königlichen Objekten manchmal neben anderen ursprünglich ägyptischen Motiven verwendet wurde. ${ }^{17}$

In der Späten Bronzezeit findet sich die Flügelsonne auf Siegeln aus dem mitannischen Nuzi und jenen der frühen mittelassyrischen Zeit, z.B. auf dem Herrschaftssiegel Šauštatars von Mitanni und dem des Eriba-Adad I. von Assyrien (vgl. Abb. 1). ${ }^{18}$

In der Residenz Hattuša führten der Wettergott und die Sonnengöttin von Arinna das Pantheon an. Sie setzten den hethitischen König ein, der ihr Land verwaltete. Die Sonnengöttin des Krönungsortes Arinna war in der Grossreichszeit höchste Instanz im Reich, war «Königin aller Länder>, verlieh dem hethitischen König ab Hattušili I. seine Legitimation. ${ }^{19}$ Die enge Verbindung zwischen König und Sonnengottheit zeigte sich ab Hattušili I. ebenfalls in der Titulatur, in welcher der König als 〈Sonne〉 u.ä. angesprochen wurde. Hattušilis’ I. Wahl der ihn legitimierenden Götter ist auch aussenpolitisch relevant: Der Wettergott spielte in den nordsyrisch-levantinischen Orten eine dominante Rolle, die Sonnengötter in universaler Sicht und gegenüber Ägypten, das ein wichtiger (Gegen-)Spieler in der Levante war. ${ }^{20}$ Die geflügelte Sonnenscheibe wurde erst mit Šuppiluliuma I. zu einem exklusiv herrschaftlichen Symbol; zuvor wurde sie auch von nicht-königlichen Personen verwendet. ${ }^{21}$

Als Echnaton den Thron bestieg, gehörte die Flügelsonne zum ikonographischen Repertoire mehrerer vorderasiatischer Könige und ihrer Eliten, die das Symbol visuell verändert und inhaltlich verwässert hatten. Ausgerechnet in der Zeit des weitesten Ausgreifens Ägyptens in den vorderasiatischen Raum war gerade dort das wichtigste ägyptische Emblem, das die Verbindung zwischen Pharao und dem universalen Sonnengott symbolisierte, nicht mehr eindeutig ägyptisch und nicht mehr eindeutig königlich besetzt. In dem für das ägyptische Königtum so fundamentalen Bereich der Beziehung Pharaos zum Sonnengott war ein Problem der Abgrenzung und Profilierung entstanden. Daher reformierte Echnaton die Beziehung zwischen ägyptischem Herrscher, Mensch und Sonnengott und unterwarf auch ihre visuelle Formulierung einer Gesamtüberholung. Er

\footnotetext{
${ }^{15}$ Pongratz-Leisten 2013: 298-299. Kaelin 2006: 130-165. Bänder 1995: 185-186.

${ }^{16}$ Polonsky 2002: 471-529. Beckman 2002: 38-39.

${ }^{17}$ Pongratz-Leisten 2013: 299-300. Eder 1999. Teissier 1996: 95-101, 196. Eder 1995: 153-154.

${ }_{18}$ Pongratz-Leisten 2013: 300-301. Yalcin 2011: 530-531. Stein 1993: nos. 653-654, 711 (Šauštatar), 729,

752, 759, 776. BERAn 1957: 144 (Eriba-Adad I.), 188-194 (Flügelsonnen), 202 (Šauštatar).

19 Pongratz-Leisten 2013: 301-305. Kutter 2008: 88-91. Klengel 1999: 322-324. Haas 1999: $178-180$.

BECKMAN 1995. HAAS 1994: 188-190.

${ }^{20}$ Zum Wettergott s. Schwemer 2001: 443-444.

${ }^{21}$ Y ALCIN 2011: 522-524.
} 
ersetzte das einst perfekt auf das ägyptische, universale, solare Königtum zugeschnittene Motiv der Flügelsonne durch die Aton-Scheibe mit Händen und erhob diese zur Darstellung der Gottheit. Atons Fürsorge galt der ganzen Welt und seine Darstellung sollte genau dies zum Ausdruck bringen.

Bei der Darstellung Atons verzichtete Echnaton bewusst auf eine anthropomorphisierte Form. Statt wie oft in Ägypten durch Addition und Synkretismus verschiedener Gottheiten eine neue zu schaffen, fokussierte man auf einen Aspekt, der allen solaren Gottheiten gemeinsam war. ${ }^{22}$ Die sichtbare Sonnenscheibe Jtn war dieser grösste gemeinsame Teiler sämtlicher solaren Götter Ägyptens und der übrigen vorderasiatischen Welt. Diese einzig real und weltweit erfahrbare Form der Sonnengottheit machte Echnaton direkt und unmittelbar zum Anbetungsobjekt und sich selbst zum einzigen Priester und Vertreter. Mit Aton und seiner Anbetung brach Echnaton mit vielen ägyptischen Traditionen: "Solar discourse was no more ... Gone was Re-Harakhty the falcon, the hprr-beetle, the Ennead with Atum at its head, Bhdty the winged sun-disc, the solar boat, the Himmelsfahrt, Apophis, myth and its use in magic, and a host of other mechanisms and images, read as referential icons." ${ }^{23}$

In den Darstellungen wurde die Sonnenscheibe Aton durch (Strahlen-)Arme und Hände in ein agierendes Wesen verwandelt. Die „emblematic personification“ war eine in der ägyptischen Ikonographie gern genutzte und spätestens seit Dynastie 0 bekannte Möglichkeit, ein Symbol durch Anfügen von Armen oder Beinen zu animieren. ${ }^{24}$

Echnatons Entscheid, den neuen Gott Aton in einer nicht-anthropomorphen Form anzubeten, war für Ägypten revolutionär, doch gab es einen solchen Trend im Vordern Orient schon länger, der vielleicht zu dem Schritt ermutig hat. Obschon in Vorderasien die Götter meist anthropomorph imaginiert und dargestellt wurden, sind sie bereits ab dem 4. Jt. v. Chr. auch über Symbole bzw. Darstellungen von Gestirnen verehrt worden. In der Mittleren Bronzezeit finden sich auf Siegeln Mesopotamiens und Kleinasiens neben anthropomorphen Darstellungen von Göttern auch Embleme für Gottheiten, z.B. die Mondsichel für den Mondgott, Blitz und Stier für Adad oder der gekrümmte Stab für Amurru. Sie wurden als Einfluss aus Syrien und der Levante gewertet, wo Kulte nicht-anthropomorpher Götter bekannt waren. ${ }^{25}$

Auf Objekten aus der Zeit der Kassiten, also einer eng mit Ägypten liierten Dynastie, war die Repräsentation von Göttern durch Symbole und Embleme verbreitet. Kassitische Rollsiegel zeigen oft eine lange Inschrift und einen Beter vor einem oder mehreren Emblemen. Frühe Beispiele dafür datieren in die Zeit von Kurigalzu I. und Burnaburiaš II. Beliebt war das Kreuz, das als Symbol für einen Gott, vielleicht sogar einen Sonnengott gedeutet wurde. ${ }^{26}$

Auf Siegeln aus dem Mitanni-zeitlichen Nuzi dominieren im Umfeld der Flügelsonne Tierkämpfe, Tiere und Bäume. Das Siegel des Šauštatar, das auch von seinen Nachfolgern verwendet wurde, zeigt eine geflügelte Sonnenscheibe, die von einem Menschen angebetet wird. ${ }^{27}$ Die Präferenz, Götter lieber als Symbole und weniger als anthropomorphe Wesen darzustellen und anzubeten, setzte sich in mittelassyrischer Zeit und darüber hinaus fort. ${ }^{28}$

\footnotetext{
${ }^{22}$ Zum Synkretismus s. HoRnung 1970: 82-90.

${ }^{23}$ REDFORD 2013: 27.

${ }^{24}$ BAINES 2004: 167.

${ }^{25}$ ORNAN 2005: 41-45

${ }^{26}$ Ornan 2005: 45-52. Stiehler-Alegria Delgado 1996: 161 (Kreuz). Seidl 1989. Beran 1957/1958: 256-258.

${ }^{27}$ STEIN 1993: 89-93 no. 711 (Šauštatar). Beran 1957: 202-203.

${ }^{28}$ ORNAN 52-59
} 

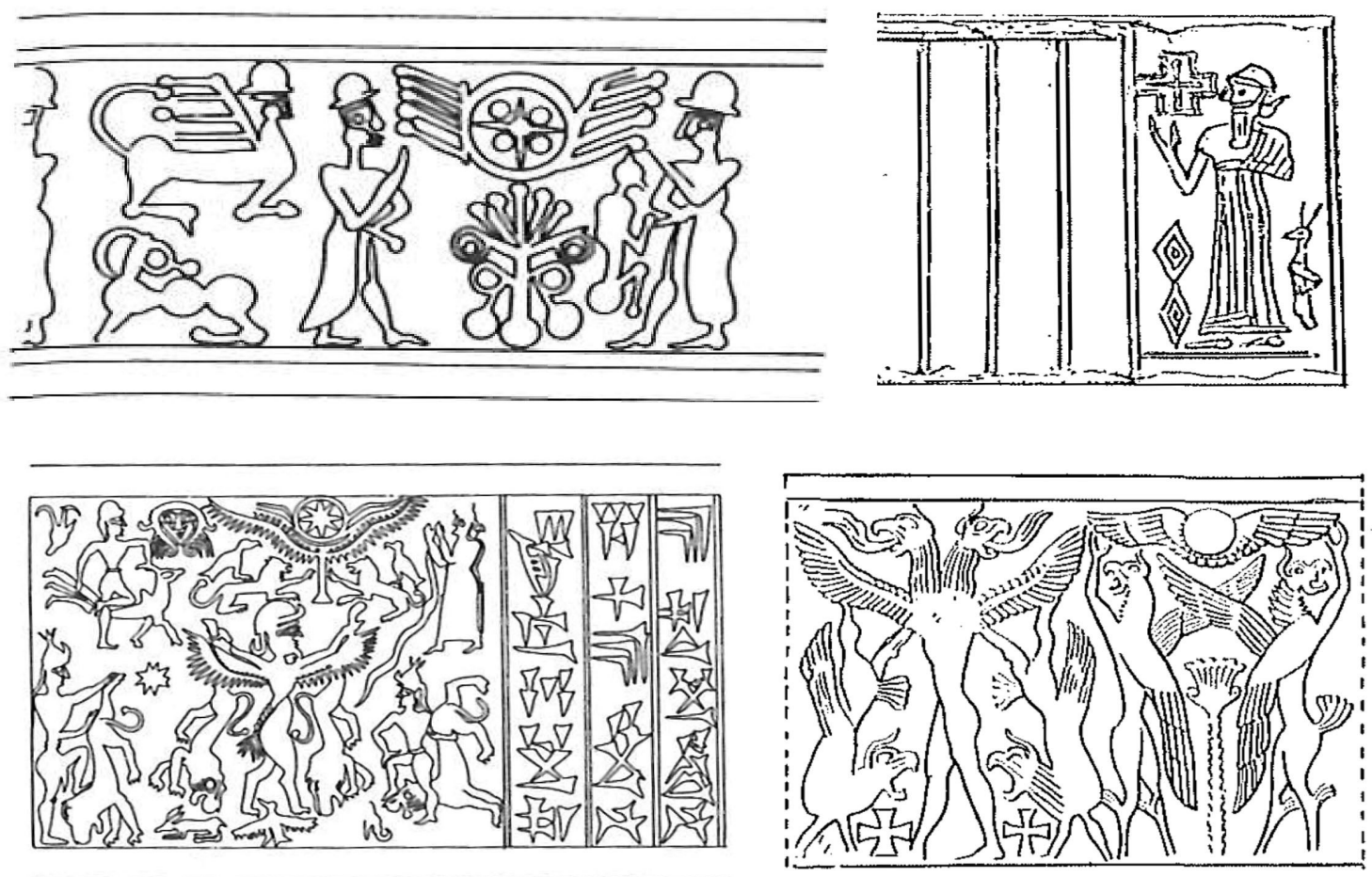

Abb. 1 Flügelsonnen und kassitisches Kreuz.

Nuzi (STEIN 1993: no. 776), Kassitisch (Zeit Kurigalzu I., STIEHLER-ALEGRIA DELGADO 1996: Nr. 52).

Šauštatar, König von Mitanni (STEIN 1993: no. 711). Eriba-Adad I von Assyrien (BERAN 1957: 144).

Mit der Darstellung seiner neuen GottheitAton als nicht-anthropomorphe Sonnenscheibe mit Händen, grenzte sich Echnaton von anderen altorientalischen Herrschern ab, die sich mit Flügelsonnen oder anthropomorphen bzw. anthropomorph gedachten Göttern zeigten. Gleichzeitig schloss er sich aber einem Trend aus Vorderasien an, eine Gottheit über eine nicht-anthropomorphe Darstellung anzubeten. Mit dem Scheitern der Amarna-Revolution verschwand jedoch die für Echnatons Regierungszeit so typische Darstellungsform der Sonnenscheibe mit Händen.

\section{Eine Residenzstadt für den Grosskönig}

$\mathrm{Ab}$ der Uruk-Zeit waren Nischenfassaden und ab der Frühen Bronzezeit (gestufte) Hochbauten höchste architektonische Auszeichnung der wichtigsten Institution altorientalischer Reiche. ${ }^{29}$ Als nächster Schritt und Steigerung entstanden in der Späten Bronzezeit ganze Residenzstädte zur Inszenierung der wichtigstes Person des Reiches. "A perfect expression of economic and ideological power, therefore, was the foundation of new residential cities. The diffusion of this idea, which was originally born out of strategic necessities, was made possible by the frequent exchange of correspondence, goods, and personages between the courts .... Once this scheme was recognized by the competing rivals, it was adapted and reinterpreted within their own cultural syntax. Thus, although the layout, the structure, and even the individual motivation for the foundation of the specific residential cities differed substantially from case to case, the idea behind it was a common one." 30

\footnotetext{
${ }^{29}$ Kaelin 2015: 131-136. Kaelin 2007. Kaelin 2006: 166-171.

${ }^{30}$ NovaK 2014: 324 (Zitat). Joffe 1998.
} 
Über den Namen wurde eine Assoziation zwischen Residenzstadt und ihrem Gründer geschaffen und an sie erinnert: Hattušili I. (,der aus Hattuša“) mit Hattuša, Kurigalzu mit DurKurigalzu („Umfassung des Kurigalzu“), Untaš-Napiriša mit Dur-Untaš („Umfassung des Untaš“ ${ }^{“}$. Auch nach der Zeit Echnatons setzte sich die Gründung neuer Residenzstädte und deren Benennung nach dem Gründerkönig fort, so Ramses II. mit Per-Ramses („Haus des Ramses“) und Tukulti-ninurta I. mit Kar-Tukulti-ninurta (,Kai des Tukulti-ninurta“) ${ }^{31}$ Taidu, im späten MitanniReich als "Stadt des Königtums" bekannt, behielt scheinbar den Namen des ursprünglichen Ortes. Vielleicht erübrigte sich die Benennung der Residenzstadt nach einem Herrscher, weil bereits der Name des Reiches Mittani "vermutlich von dem Namen eines frühen Herrschers abgeleitet und damit primär politischer, nicht ethnischer oder geographischer Natur" war. ${ }^{32}$

In mindestens drei Fällen befreien Texte und Inschriften den für die Residenzstadt gewählten Ort von jeglichen älteren Ansprüchen durch andere Herrscher oder Götter. Hattušili I. gründete seine Stadt Hattuša an einem Ort, den Anitta einst unbesiedelbar gemacht hatte. Echnaton fand auf Befehl Atons einen Ort, der keiner anderen Gottheit gehörte. Und später baute Tukulti-Ninurta I., auf göttlichen Befehl, an einem Ort, an dem angeblich noch nie gebaut worden war. Die Grosskönige mussten klarstellen, dass sie für ihre Residenzstadt niemandem, erst recht keiner Gottheit etwas wegnahmen (s.u.).

Mit Hattuša machte Hattušili I. einen Ort zu Residenz, Staatsverwaltung und Kultzentrum (etwa 17. Jh. v. Chr.), den rund hundert Jahre früher König Anitta zerstört, unbesiedelbar gemacht und dem Vergessen überlassen hatte: "An ihrer Stelle aber sä[te] ich Unkraut. Wer immer aber nach mir König wird und Hattuša wiederbesiedelt, den [soll] der

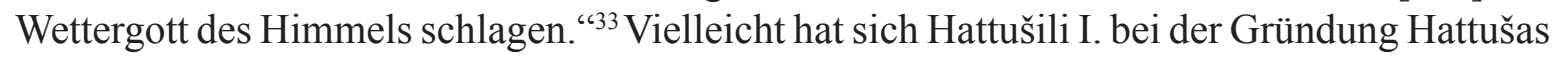
durch Sargon von Akkad und dessen neu gegründeter Hauptstadt Agade inspirieren lassen. Das syrisch-mesopotamische Schreiberwesen, das zur Verwaltung des hethitischen Reiches eingeführt wurde, brachte nämlich auch historisches Wissen um die grossen Leistungen früherer Herrscher mit sich. Als Hattušili I. den Euphrat überquerte, verglich er sich jedenfalls explizit mit Sargon von Akkad: „No one had crossed the Euphrate River, but I, the Great King, the Tabarna, crossed it on foot, and my army crossed it on foot behind me. Sargon (of Akkad also) crossed it. [He] fought the troops of Hahha, but [he] did not do anything to Hahha. He did not burn it down; smoke was not visible to the stom-god of Heaven. But I, the Great King, the Tabarna, destroyed Haššuwa and Haḩha, and [burned] them down with fire. I showed smoke to the sun-god of Heaven and the storm-god ..." ${ }^{34}$ Hattušili I. betont also, dass er Sargon übertrifft, da er, im Gegensatz zu jenem, die Stadt Haḩha zerstörte. Ähnliches Wettbewerbsdenken mag bei der Gründung der Residenzstadt Hattuša hineingespielt haben.

Im hethitischen Alten Reich umfasste die Stadtmauer Hattušas, der „Stadt der Götter“, einen Palastbezirk mit Totentempel, sowie Tempelbauten mehrerer Gottheiten des Reiches, darunter natürlich für den Wettergott und die Sonnengöttin von Arinna. ${ }^{35}$ Mit seinem Namen Hattušili I. (,,der aus Hattuša“) stellte der König eine enge Verbindung zu seiner Hauptstadt her. Damit trat er eine Modewelle los, die in den folgenden Jahrhunderten sämtliche Königtümer der altorientalischen Grossmächte erfasst.

Hattušilis' Nachfolger Muršili I. überfiel Babylon, was die Machtergreifung durch die Dynastie der Kassiten ermöglichte. ${ }^{36}$ Mit Karaindaš nahmen die Kassiten politische

\footnotetext{
${ }^{31} \mathrm{Zu}$ den Stadtnamen NovaK 1999: 381-384.

${ }^{32}$ RLA s.v. Mittan(n)i § 4 (Zitat).

${ }^{33}$ Schachner 2011: 69-81. TUAT NF 2 (Klinger): 140-141 (Zitat). Klengel 1998: 43-44; Neve 1993: 8.

${ }^{34}$ Beckman in Chavalas 2006: 221 (Zitat). Klengel 1998: 51-52, 55.

${ }^{35}$ HaAs 1994: 616-620. Bittel 1983: 59ff., 92 (Plan). RlA s.v. Hattuša.

${ }^{36}$ KLENGEL 1998: 56, 64-66.
} 
Beziehungen mit Ägypten auf. Sein Enkel Kurigalzu I. pflegte diese weiter, bekam von Vorfahren Echnatons sogar Gold geliefert, was seine Bauprojekte sicher förderte. Kurigalzu I. gründete und benannte nach sich selbst die Residenzstadt Dur-Kurigalzu „Umfassung des Kurigalzu“ (Aqar Quf, ca. 14. Jh. v. Chr.). ${ }^{37}$ Bekannt sind hier ein Palast, die Ziqqurat und die Tempel, die Enlil, Ninlil und Ninurta geweiht waren. Auch hier bekamen die wichtigsten Götter des Landes eine Dependance. Dass Kurigalzu sich ebenfalls eine Residenz bauen liess, war wohl nicht einzig geostrategisch bedingt, sondern diente auch der Demonstration politischer Gleichwertigkeit zu den Hethitern. ${ }^{38}$

Untaš-Napiriša von Elam war mit den Herrschern von Babylon familiär verbunden. Seine Mutter war vielleicht Tochter von Kurigalzu I., seine Frau vielleicht Tochter von Burnaburiaš II., einem Zeitgenossen Amenophis III. und Echnatons ${ }^{39}{ }^{39}$ Auch Untaš-Napiriša errichtete mit Al-Untaš-Napiriša bzw. später Dur-Untaš-Napiriša, „Umfassung des Untasch“ (mod. Tschoga Zanbil), eine Residenz, die nach ihm benannt war. Eine vier Kilometer lange Mauer umgibt ein Areal von etwa 100 ha. Im Zentrum der Anlage und innerhalb einer kleineren Umfassungsmauer stand eine Ziqqurat, die den Göttern Napiriša und Inšušinak gewidmet war, sowie weitere Tempel und Schreine von etwa 25 verschiedenen Göttern. Im Stadtareal standen mehrere grosse Gebäude, eines davon über Grabgewölben. Zum östlichen Stadttor soll eine "Prozessionsstrasse" geführt haben. ${ }^{40}$

In Ägypten war während der 18. Dynastie der Amun-Tempel von Karnak wichtigster Kultort und Theben Hauptstadt. Hier wurde auch in den ersten Jahren Echnatons ein grosser Tempel für Aton errichtet. Die "plötzliche" Gründung und der Wegzug nach Achet-aton wirken innerägyptisch "revolutionär", doch der Bau von Achet-Aton als Atons und Echnatons neuer Hauptstadt steht mit Hattuša, Dur-Kurigalzu und Dur-Untaš in der Traditionslinie der Residenzneugründungen altorientalischer Grosskönige der Späten Bronzezeit. Letztere waren eng mit ihrem jeweiligen Erbauer verbundene Kult- und Residenzstadt, in denen mehrere Götter aus dem ganzen Reich repräsentiert und einen Tempel hatten. Wie andere, neu gegründete Residenzstädte Vorderasiens diente auch Achet-Aton der Inszenierung des Königs und der Beherbergung des wichtigsten Kultes des Reiches. Nur war halt zu jenem Zeitpunkt in Ägypten gerade Aton einziger Gott.

Auf halber Strecke zwischen Memphis und Theben, auf einem Wüstenplateau am östlichen Nilufer, entstand die Residenzstadt Achet-Aton (mod. Tell el-Amarna). Dieser "Horizont des Aton" wurde klar begrenzt, die Aussengrenzen mit Stelen markiert. Nach dem Wortlaut dieser Grenzstelen hatte Aton selbst zum Ort geführt, der zuvor unbesiedelt und von niemandem beansprucht war, und den Auftrag zur Neugründung erteilt: „Behold Aten! The Aten wishes to have [something] made for him as a monument with an eternal and everlasting name. Now it is the Aten, my father, who advised me concerning it, (namely) Akhet-Aten. No official had ever advised me concerning it, nor had any people in the entire land ever advised me concerning it, to tell me [a plan] for making Akhet-Aten in this distant place. It was the Aten, my father, [who advised me] concerning it, so that it could be made for him as AkhetAten. Behold, I did not find it provided with shrines or plastered with tombs or porticoes (?) ... or covered with ... (or) with the remnant of anything which had happened to it, so that it was not [ .. . ing] me ... Akhet-Aten for the Aten, my father. Behold, it is pharaoh, 1.p.h., who found it, when it did not belong to a god, nor to a goddess; when it did not belong to a male

\footnotetext{
${ }^{37}$ Rainey 2015: EA 9 und 11. BrinKman 1976: 205-246.. RLA s.v. Kurigalzu.

${ }^{38}$ NovaK 1999: 85-90.

${ }^{39}$ Rainey 2015: EA 6-14. Potts 2014: 27-29. Aber vgl. Roaf 2017: 186-189. Mofidi Nasrabadi 2018a: $234-236$.

${ }^{40}$ Mofidi Nasrabadi 2018a: 241. Mofidi Nasrabadi 2018b: 521-528. Potts 1999: 222ff.
} 
ruler, nor to a female ruler; when it did not belong to any people to do their business with it. [Its ... ] is not known, ( but) I found it widowed ..." "441

In den Felsen, die das Wüstenplateau im Osten begrenzen, liegen die Gräber des Königs, seiner Familie und Beamten. Die Stadt hatte mehrere Aton gewidmete Tempel und Bauten. Die königliche Prozessionsstrasse bildete die Hauptachse der Stadt und verband den nördlichen und den südlichen Palast. Der grosse und der kleine Aton-Tempel waren die beiden Hauptkultgebäude, in denen Aton, in gegen den Himmel offenen Höfen, die mit zahlreichen Altären ausgestattet waren, mit seinen Strahlenhänden direkt auf seine Opfer zugreifen konnte. Echnaton und seine Familie wurden als wichtigste und meist einzige Funktionäre des Kultes dargestellt. ${ }^{42}$

Achet-Aton war nicht nur die Residenz Echnatons sondern auch jene Atons, des universalsten Sonnengottes der altorientalischen Welt. Hier wurde er von Echnaton (und seiner Familie) verehrt, wobei der König auch als sein alleiniger irdischer Vertreter und sein einziges Sprachrohr galt. Der Name der Stadt Achet-Aton, "Horizont des Aton", schuf wie bei den vorderasiatischen Grosskönigen durch das Element "Aton" eine Verbindung zwischen der Residenz und ihrem Auftraggeber. Das andere Element mesopotamischer Residenzgründungen "Dur" "Umfassung, Ummauerung" wurde durch "Horizont, Lichtland" sogar übertroffen, denn jenseits einer konkreten geographischen Stadtbegrenzung markiert der Horizont die ultimative Grenze, Ummauerung, Umfassung der Erde. Während die vorderasiatischen Herrscher in ihrer Residenz die Grösse ihres eigenen Königtums durch eine Vielfalt an Göttern und Tempeln demonstrierten, zelebrierte Echnaton in seiner neue Hauptstadt seinen zum "König der Welt" erhobenen Sonnengott in seiner omnipräsentesten, essentiellsten und unmittelbarsten Form, nämlich als die Sonne selbst.

\section{Nachwirkung}

Die Inszenierung von Echnatons neuem Gott Aton schloss sich einerseits Trends aus Vorderasien an, regte andererseits dort aber auch Innovationen an.

In Hatti wurde unter Šuppiluliuma I. die geflügelte Sonnenscheibe, die zuvor auch von nicht-königlichen Personen verwendet wurde, zu einem exklusiv herrschaftlichen Symbol. Diese geflügelte Sonnenscheibe in der frühesten Fassung der hethitischen Ädicula war direkt von ägyptischen Vorbildern inspiriert. ${ }^{43}$ Šuppiluliuma I. regierte spätestens zur Zeit von Echnatons unmittelbaren Nachfolgern, also in einer Zeit, in der die Sonnenscheibe mit Händen und die geflügelte Sonnenscheibe als wichtigstes Logo des ägyptischen Königs in Konkurrenz standen. Dies und ihre Verbreitung in Syrien mag die Adoption der geflügelten Sonnenscheibe als königliches Logo durch den hethitisches König begünstigt haben.

Unter Muwatalli II. wurde die Hauptstadt für kurze Zeit nach Tarhuntassas versetzt, er selbst nannte sich "Grosser König von Tarhuntassas”. „In the wake of a comprehensive religious reform, which may perhaps be best compared to Akhenaton's reform in Egypt, he moved the imperial seat from the centuries-old capital of Hattusa to a previously unknown palace in the Lower Land, Tarhuntassa. “44 Auch dieses Experiment könnte also von Echnatons Aktivitäten angeregt worden sein.

Einen weiteren möglichen Versuch von Hybris finden wir in Tall al-Hamidiya/Taidu? (NO-Syrien). Die Stadtarchitektur kann eigentlich nur als Neugründung einer wichtigen

\footnotetext{
${ }^{41}$ Murnane 1995: $75-77$ (Zitat). Wilkinson 2000: 140; Arnold 1992: 178ff. Joffe 1998: 552-556.

${ }^{42}$ SteVens 2016. Hoffmeier 2015: 136-164. Hornung 2003: 69-96.

${ }^{43}$ YALCIN 2011. DinCOL 2002. BÖRKER-KLÄHN 1993.

${ }^{44}$ BRYCe 2005: 230-233. Singer 2001: 395 (Zitat).
} 
Residenz interpretiert werden. Der alte Hügel wurde zu einer Terrasse geebnet, auf der drei grosse Baukomplexe enstanden: der kaum ergrabene Ost-Bereich, wo die Tempel vermutet werden; der SW-Palast, wo Texte und Abrollungen eine administrative Funktion nahelegen, und der repräsentative Zentral-Palast, ein vier-terrassiger Stufenbau mit drei monumentalen Zugangstreppen, auf dessen oberster Terrasse eine Palastanlage stand. Keramik, Siegel und Texte datieren die Anlage zumindest in die Mitanni-Zeit. ${ }^{45}$ Der Mitanni-Herrscher, der nie ausdrücklich Göttlichkeit für sich beanspruchte, säkularisierte damit den (ziqqurat-artigen) Stufenbau, eine im Vordern Orient den Göttern vorbehaltene Bauform. In vorderasiatischer Tradition und Perspektive irritiert diese Verwendung einer sakralen Bauform für den Herrscher. Sie wirkt jedoch weniger befremdlich aus einer ägyptischen Perspektive, das über Generationen eng mit Mitanni verbunden war. Die Grundidee der Anlage zeigt nämlich auch Parallelen zu den Totentempeln von Mentuhotep und Hatschepsut in Deir el-Bahari: lange Zugangstreppen, mehrere Terrassen, durch Pfeiler(vorsprünge) strukturierte Fassaden. Auf der obersten Terrasse stand der wichtigste Bau des Herrschers - in Ägypten der Totentempel, im Vorderen Orient der Palast. Der Zentralpalast von Tall al-Hamidiya übertrifft das ägyptische Vorbild allerdings in der Breite der Treppen und deren Anzahl. Die Nachahmung wäre dadurch erleichtert worden, dass seit Hatschepsut kein ägyptischer Herrscher Interesse für diese Art von gestuftem Terrassenbau gezeigt hatte. Falls der Mitanni-Herrscher sich aus Ägypten inspirieren liess, wäre seine Anlage die Nachahmung eines Baus für Könige und weniger eines Baus für Götter, selbst wenn bei ägyptischen Totentempeln die Grenzen zwischen König und Göttern sehr viel fliessender sind. In Vorderasien blieb die Anlage allerdings einmalig.
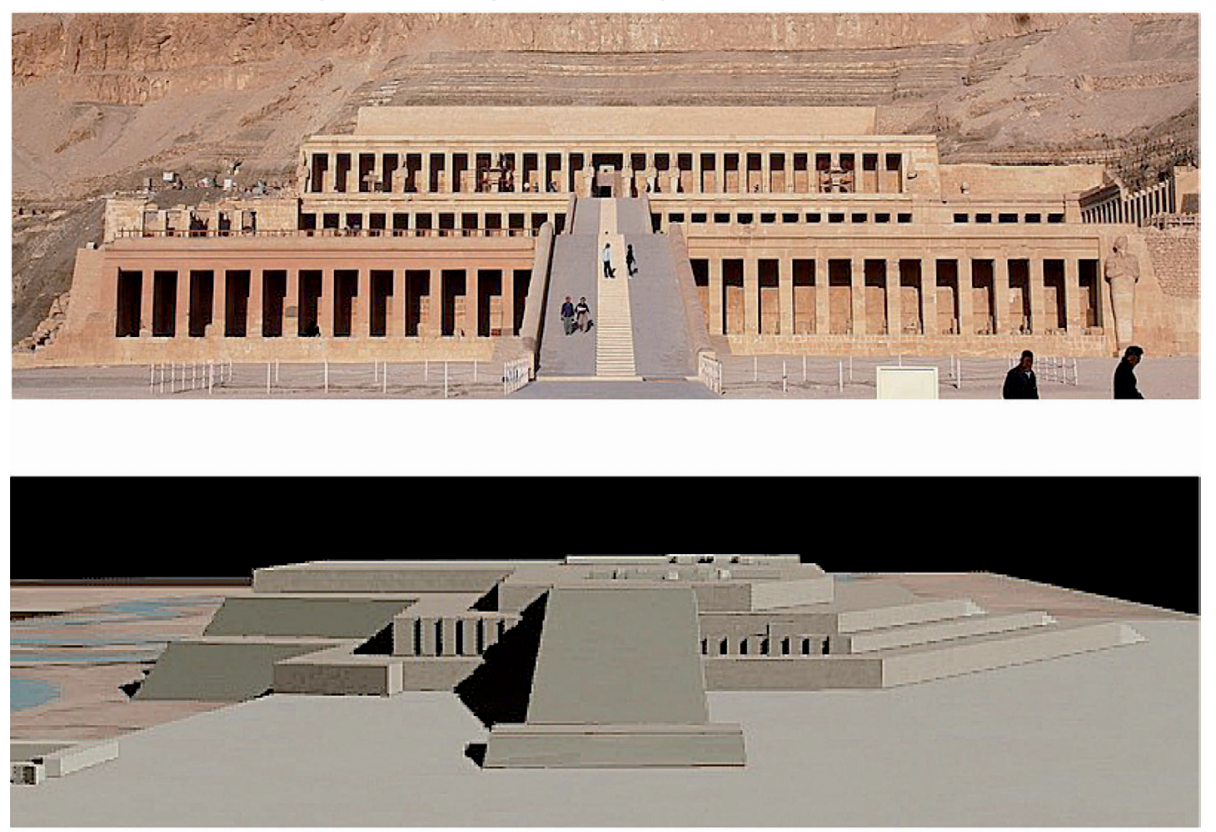

Abb. 2 Herrscherbauten mit langen Zugangstreppen, mehreren Terrassen und durch Pfeiler(vorsprünge) strukturierten Fassaden.

Totentempel der Hatschepsut, Deir el-Bahari (CCreative Commons https://commons. wikimedia.org/wiki/File:Deir_el-Bahari_0489.JPG) vs. Zentralpalast von Tall al-Hamidiya

(Detail aus der 3D Rekonstruktion (C) Gino Caspari (https://sites.google.com/sitel

hamidiyataidu/home/periods-of-settlement/the-mitanni-period/the-residential-terrace).

\footnotetext{
${ }^{45}$ Kessler 2014. Kaelin 2013. WÄfler 2007. Wäfler 2003:17-79. Die früheste gesicherte Erwähnung von Taidu als Hauptstadt finden wir bei Adad-Nirari I. (MAYER 1986: 233-236).
} 
Bei der nächsten Residenzstadt Ägyptens orientierte man sich am konventionellen vorderasiatischen Modell. Ramses II. liess im östlichen Nildelta (Qantir, 13. Jh. v. Chr.) seine Residenzstadt Per-Ramses genauer „Haus-des-Ramses-gross-an-Macht“ bauen, ausgestattet mit Palast und Tempeln für traditionelle ägyptische und vorderasiatische Gottheiten. ${ }^{46}$ Akzeptiert waren politisch-religiös korrekte Residenzstädte, die zwar eng mit dem Königtum verbunden waren, aber auch die Götter im Reich repräsentierten.

Daran hielt sich auch Tukulti-Ninurta I., der - kaum war Assyrien in die Liga der Grosskönige aufgestiegen - seine Residenzstadt "Kar Tukulti-Ninurta" (Tulul al-Aqar, 13. Jh. v. Chr.) gründete. ${ }^{47}$ Ähnlich wie im Falle der Texte auf Echnatons Grenzstelen betonte Tukulti-ninurta I. den direkten Auftrag seines Gottes Assur sowie die Verlassenheit und Unbewohntheit des Gründungsortes: „At that time the god Assur, my lord, requested of me a cult centre on the bank opposite my city, the desired object of the gods, and he commanded me to build his sanctuary. At the command of the god Assur, the god who loves me, I built before my city, Assur, a city for (the god) Assur on the opposite bank, besides the Tigris, in uncultivated plains and meadows where there was neither house nor dwelling, where no ruin hills of rubble had accumulated, and no bricks had been laid. I called it Kar-Tukulti-Ninurta." Eine Inspiration des Topos aus Ägypten liegt nahe, zumal auch das Tukulti-ninurta-Epos, eine literarische Zelebration des assyrischen Königs, stark von Ägypten beeinflusst war. ${ }^{48}$

Ein Nachhall der atonischen Ikonographie findet sich auf dem assyrischen "Broken Obelisk". In einer Reihe von Göttersymbolen steht über dem Herrscher erstmals eine agierende geflügelte Sonne. Diese geflügelte Sonne hat zwei menschliche Hände: eine offene Hand weist auf den König, eine geschlossene Hand hält einen Bogen und Pfeile. Diese Bildidee, nach welcher der König von der Sonne Waffen erhält, findet sich auch unter den frühen Darstellungen Echnatons, der zum Erschlagen der Feinde Krummschwert und Keule, die traditionellen Waffen Pharaos, zugereicht bekommt. Wie Echnatons Aton wurde auch auf dem "Broken Obelisk" das Symbol der Sonne mittels von ihr ausgehenden Händen animiert und handlungsfähig gemacht. ${ }^{49}$ Doch diese einfache Form der Animierung durch Anthropomorphisierung scheint in Assyrien für Befremdung gesorgt zu haben, denn die Flügelsonne mit Hand blieb nicht im Repertoire. Die Animierung der geflügelten Sonne wurde in Assyrien und später in Persien mit der Figur in der Sonnenscheibe erreicht. Den assyrischen Seh- und Verständniskonventionen war die Animation eines Symbols oder Objektes durch einfache Addition von Körperteilen wohl schlicht zu fremd, weshalb man die Flügelsonne durch Hinzufügung einer menschlichen Figur zu Interaktion und Handlungsmacht befähigte. Visuell schuf man, unabhängig davon welche Gottheit dieses Ensemble von Flügelsonne und Menschenfigur nun genau repräsentieren soll, einen Bezug zwischen König und Sonne. ${ }^{50}$ Dieser Bezug ist auch in der Keilschrift zu finden: das Zeichen "MAN" bzw. "20" kann sowohl für "König" als auch für den Sonnengott Šamaš stehen. ${ }^{51}$

\footnotetext{
${ }^{46}$ WiLKINSON 2000: 108. ARNOLD 1992: 211.

${ }^{47}$ Novak 1999: 120-128. Dolce 1997. Eickhoff 1985. RlA s.v. Kar Tukulti-Ninurta.

${ }^{48}$ Deller et al. 1994: 467 (Zitat). Zum Tukulti-ninurta-Epos s. Kaelin 2016.

${ }^{49}$ Pongratz-Leisten 2013: 305-308. Curtis 2007. Ornan 2007. Morenz 2003: 130-137. Börker-Klähn 1982:

178, Nr. 131. TRAUNECKER 1984: 60-61.

${ }^{50}$ Zur Figur in der Flügelsonne s. GARRISON 2009: 36-40.

${ }^{51}$ Borger 1986: Nr. 471.
} 

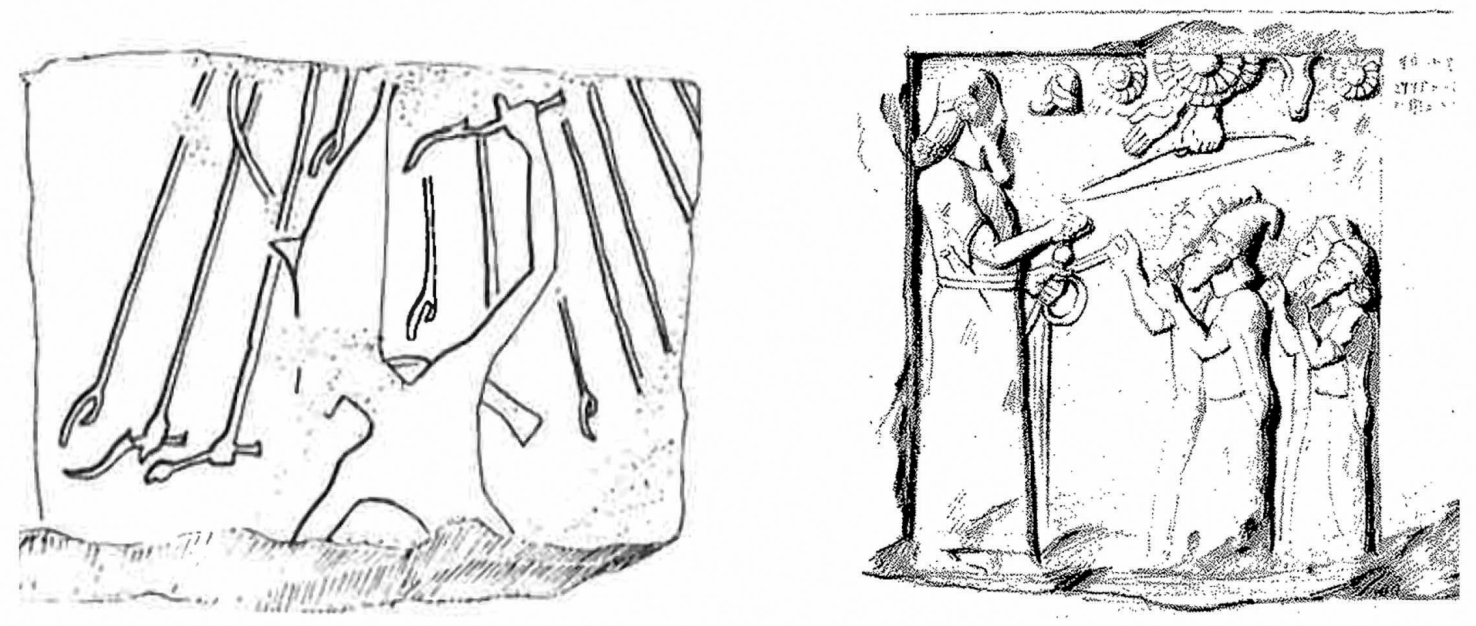

Abb. 3 Der König erhält Waffen von einer mit Händen animierten Sonne. Echnaton (TRAUNECKER 1984: 61) vs. Broken Obelisk (CURTIS 2007: 54).

Echnatons Revolution endete mit seinem Tod. Tutanchamun restaurierte schnell das alte System. Echnatons Tempel und Residenzstadt wurden später abgebaut, das Baumaterial wiederverwendet. Der Scheibenaton wurde aufgegeben. ${ }^{52}$ Die "Amarna-Religion" war nicht nur für innerägyptische Verhältnisse ein Wagnis, sondern auch gegenüber den anderen Grossreichen eine Provokation. Schliesslich masste sich Echnaton an, einzige direkte Kontaktperson und einziges Sprachrohr einer in allen vorderasiatischen Kulturen wichtigen Gottheit zu sein. Die Grosskönige strebten über Diplomatie und Politik gleichwertige und gegenseitige Anerkennung an. Titel, Anreden, Residenzen und solare Aspekte waren Elemente davon. Echnaton erhob aber seinen Aton und damit sich selbst über die (Sonnen-) Götter der anderen Reiche, negierte den Polytheismus und störte zumindest ideologisch die Legitimation anderer Herrscher und damit das Gleichgewicht der Mächte. "Akhenaten, in a word, consigned to the rubbish heap far more than he created." 53

\section{BIBLIOGRAPHIE}

ÁLVAREZ-MON, J. ET AL.,

2018 The Elamite World. London.

ARNOLD, D.

1992 Die Tempel Ägyptens. Götterwohnungen - Baudenkmäler - Kultstätten. Zürich.

ARUZ, J. ET AL.

2013 Cultures in Contact. From Mesopotamia to the Mediterranean in the Second Millennium B.C. Metropolitan Museum of Art Symposia. New York.

ASSMANN, J.

1984 Ägypten. Theologie und Frömmigkeit einer frühen Hochkultur. Stuttgart.

\footnotetext{
${ }^{52}$ Hoffmeier 2015: 238-244. Hornung 2003: 115-138

${ }^{53}$ REDFORD 1976: 47.
} 
BÄNDER, D.

1995 Die Siegesstele des Naramsîn und ihre Stellung in Kunst- und Kulturgeschichte. Idstein.

BAINES, J.,

2004 “The Earliest Egyptian Writing: Development, Context, Purpose". Houston, S. D. (ed.), 2004. The First Writing: Script Invention as History and Process. Cambridge, pp. 150-189.

BARTELMUS, A. - STERNITZKE, K. (EDS.)

2017 Karduniaš. Babylonia under the Kassites. The proceedings of the symposium held in Munich, 30 June to 2 July 2011. Tagungsbericht des Münchner Symposiums, 30. Juni bis 2. Juli 2011. 2 vol.. Boston.

BAYER, C.

2007 Sonnenhymnen. Ägyptisch/Deutsch.. Stuttgart.

BECKMAN, G

1995 Royal Ideology and State Administration in Hittite Anatolia. CANE 1, 529-543.

2002 "My Sun-God". Reflections of Mesopotamian Conceptions of Kingship among the Hittites. Panaino/Pettinato 2001, pp. 37-44.

BERAN, T.

1957 Assyrische Glyptik des 14. Jahrhunderts. ZA 52, pp. 141-215.

1957-1958 «Die babylonische Glyptik der Kassitenzeit». Archiv für Orientforschung 18 , pp. $255-278$.

BORGER, R.

1986 Assyrisch-babylonische Zeichenliste. Alter Orient und Altes Testament 33.

BÖRKER-KLÄHN, J.

1982 Altvorderasiatische Bildstelen und vergleichbare Felsreliefs. Baghdader Forschungen 4. Mainz.

1993 «Vivat Rex!». Syria 70.1-2, pp. 99-108.

BRINKMAN, J. A.

1976 A Catalogue of Cuneiform Sources Pertaining to Specific Monarchs of the Kassite Dynasty. Materials and Studies for Kassite History 1. Chicago.

2017 "Babylonia under the Kassites: Some Aspects for Consideration". Bartelmus/ Sternitzke 2017, pp. 1-44.

BRYCE, T.

2005 The Kingdom of the Hittites. Oxford.

CHAVALAS, M. W. (ed.)

2006 The Ancient Near East: historical sources in translation. Blackwell sourcebooks in ancient history. Malden, Mass.. 
CURTIS, J.,

2007 “The Broken Obelisk” Iraq 59, pp. 53-56.

DELLER, K. ET AL.

1994 "Two New Royal Inscriptions Dealing with Construction Work in Kar-TukultiNinurta”. Baghdader Mitteilungen 25, 459-472.

DINCOL, A.

2002 „Tabarna“ und „Ädikula“-Siegel. Die Siegel hethtischer Grosskönige und Grossköniginnen. In: Die Hethiter und ihr Reich. Das Volk der 1000 Götter. Kunst- und Ausstellungshalle der Bundesrepublik Deutschland, Bonn, pp. 88-93.

DOLCE, R.

1997 "The City of Kar-Tukulti-Ninurta. Cosmic Characteristics and Topographical Aspects”. Assyrien im Wandel der Zeiten, 39. RAI 1992. HSAO 6. S. pp. 251-258.

DUISTERMAAT, K. - REGULSKI, I. (eds.)

2011 Intercultural contacts in the ancient Mediterranean. Proceedings of the International Conference at the Netherlands-Flemish Institute in Cairo, 25th to 29th October 2008. Orientalia Lovaniensia analecta 202. Leuven.

EDER, CHR.

1995 Die ägyptischen Motive in der Glyptik des östlichen Mittelmeerraumes zu Anfang des 2. Jts. v. Chr. Orientalia Lovaniensia Analecta 71. Leuven.

1999 «Einfluss ägyptischer Königsikonographie in der Levante zu Anfang des 2. Jahrtausends v. Chr.». Gundlach/Seipel 1999, pp. 125-154.

EICKHOFF, T.

1985 Kar Tukulti-Ninurta. Eine mittelassyrische Kult-und Residenzstadt. Abhandlungen der Deutschen Orient-Gesellschaft 21.

FELDMAN, M. H.

2006 Diplomacy by Design: Luxury Arts and an „International Style“ in the Ancient Near East, 1400-1200 BCE. Chicago.

GARRISON, M. B.

2009 Visual Representation of the Divine and the Numinous in Early Achaemenid Iran: Old Problems, New Directions. Eggler J./Uehlinger Ch., eds., Iconography of Deities and Demons in the Ancient Near East, http://www.religionswissenschaft.uzh.ch/idd/ prepublications/e_idd_iran.pdf (7.6.2018).

GESTOSO SINGER, G. N.

2006 "El intercambio de materias primas y bienes de prestigio entre Egipto y Mesopotamia (siglos XV y XIV a.C.)" Aula Orientalis 24, pp. 189-212.

GÖRG, M.

2007 Ägyptische Religion: Wurzeln - Wege - Wirkungen . Religionen in der Umwelt des Alten Testaments 3. Kohlhammer-Studienbücher. Theologie 4,3. Stuttgart. 
GUNDLACH, R.

1998 Der Pharao und sein Staat. Die Grundlegung der ägyptischen Königsideologie im 4. und 3. Jahrtausend. Darmstadt.

GUNDLACH, R. - SEIPEL, W.

1999 Das frühe ägyptische Königtum. Akten des 2. Symposiums zur ägyptischen Königsideologie in Wien, 24.-26.9.1997. Beiträge zur altägyptischen Königsideologie 2; Ägypten und Altes Testament 36,2. Wiesbaden.

HAAS, V.

1994 Geschichte der hethitischen Religion. Handbuch der Orientalistik I/15. Leiden.

1999 «Das hethitische Königtum. Gundlach/Seipel»1999, pp. 171-198.

HEINZ, M. - FELDMAN, M. H.

2007 Representations of Political Power. Case Histories from Times of Change and Dissolving Orders in the Ancient Near East. Winona Lake.

HOFFMEIER, J. K.,

2015 Akhenaten and the Origins of Monotheism. Oxford/New York.

HORNUNG, E.

1970 Der Eine und die Vielen. Ägyptische Gottesvorstellungen. Mainz.

2003 Echnaton. Die Religion des Lichts. Düsseldorf.

HORNUNG, E. ET AL. (eds.)

2006 Ancient Egyptian Chronology. Handbook of Oriental Studies. Sect. 1, The Near and Middle East, Vol. 83. Leiden.

JOFFE, A. H.

1998 "Disembedded Capitals in Western Asian Perspective". Comparative Studies in Society and History 40, pp. 549-580.

KAELIN, O.

2006 „Modell Ägypten“: Adoption von Innovationen im Mesopotamien des 3. Jahrtausends v. Chr. Orbis Biblicus et Orientalis, Series Archaeologica 26. Fribourg.

2007 «Ägypten als Modell für mesopotamisches «hoch bauen» - akkadisch zaqāru(m) und ägyptisch sq3j als sprachliches Indiz». Hefte des Archäologischen Seminars der Universität Bern 20, pp. 59-64.

2013 «Tall al-Hamidiya/Ta'idu?». Bonatz, D. /Martin, L. (Hgg.). 100 Jahre archäologische Feldforschungen in Nordost-Syrien - eine Bilanz. Wiesbaden, pp. 181-192.

2015 Ein vorderasiatisches Ideen-Cluster zur frühen Inszenierung des ägyptischen Herrschers. Schmidt 2015, pp. 127-148.

2016 Das Tukultī-Ninurta-Epos und das ägyptische Qadě̌-Schlacht-Poem. Ideentransfer in der Inszenierung eines assyrischen Herrschers. Jenni, H./Saur, M. (Hgg.). Nächstenliebe und Gottesfurcht. Beiträge aus alttestamentlicher, semitistischer und altorientalistischer Wissenschaft für Hans 
KESSLER, K.

2014 «Neue Tontafelfunde aus dem mitannizeitlichen Taidu - ein Vorbericht». Bonatz, D. (ed.), The Archaeology of Political Spaces. The Upper Mesopotamian Piedmont in the Second Millennium BC. Berlin/Boston, pp. 35-42.

KLENGEL, H.

1998 Geschichte des Hethitischen Reiches. Handbuch der Orientalistik I/34. Leiden.

KLINGER, J.

2006 Chronological Links between the Cuneiform World of the Ancient Near East and Ancient Egypt. Hornung et al. 2006, 304-324.

KUTTER, J.

2008 nūr ilī. Die Sonnengottheiten in den nordwestsemitischen Religionen von der Spätbronzezeit bis zur vorrömischen Zeit. Alter Orient und Altes Testament 346. Münster.

LÄ. HELCK, W. /WESTENDORF, W. (HGG.)

1975ff. Lexikon der Ägyptologie. 7 Bde. Wiesbaden.

MAYER, W.

1986 «Taide oder Waššukanni? Name und Lage der Hauptstadt Mitannis». UgaritForschungen 18, pp. 231-236.

MILLER, J.

2017 "Political Interactions between Kassite Babylonia and Assyria, Egypt and Hatti during the Amarna Age". Bartelmus/Sternitzke 2017, pp. 93-111.

MOFIDI NASRABADI, B.

2018 "Elam in the Middle Elamite Period". Álvarez-Mon et al. 2018, pp. 232-248.

2018 "Elamite Architecture". Álvarez-Mon et al. 2018, pp. 507-530.

MORENZ, L. D.

2003 „Zur Ikonographie des Broken Obelisk und möglichen Beziehungen zu Altägypten. Eine Detailstudie interkultureller Ideen- und Bildkontakte in der zweiten Hälfte des 2. Jt. v. Chr.» In: Morenz, L. D. - Bosshard-Nepustil, E., 2003. Herrscherpräsentation und Kulturkontakte Ägypten - Levante - Mesopotamien. Acht Fallstudien. Alter Orient und Altes Testament 304. Münster, pp. 125-144.

MURNANE, W. J.

1995 Texts from the Amarna Period in Egypt. Atlanta, GA.

MURNANE, W. J. - VAN SICLEN III, C. C.

1993 The Boundary Stelae of Akhenaten. London/New York.

NEVE, P.

1993 Hattuša. Stadt der Tempel und Götter. Mainz. 
NOVÁK, M.

1999 Herrschaftsform und Stadtbaukunst: Programmatik im mesopotamischen Residenzstadtbau von Agade bis Surra man ra'ā. Schriften zur vorderasiatischen Archäologie 7. Saarbrücken.

2014 "The Phenomenon of Residential Cities and City Foundations in the Ancient Near East: Common Idea or Individual Cases?”. Osborne 2014, pp. 311-332.

OEAE. REDFORD, D. B. (ED.)

2001 The Oxford Encyclopedia of Ancient Egypt. 3 vols. Oxford.

ORNAN, T.

2005 The Triumph of the Symbol. Pictorial Representation of Deities in Mesopotamia and the Biblical Image Ban. Orbis Biblicus et Orientalis 213. Fribourg.

2007 "Who is Holding the Lead Rope? The Relief of the Broken Obelisk". Iraq 69, pp. 59-72.

OSBORNE, J. F. (ed.)

2014 Approaching Monumentality in the Archaeological Record. New York.

PODANY, A. H.

2010 Brotherhood of Kings. How International Relations Shaped the Ancient Near East. Oxford.

POLONSKY, J.

2002 The Rise of the Sun God and the Determination of Destiny in Ancient Mesopotamia. UMI Dissertation Services. Ann Arbor.

PONGRATZ-LEISTEN, B.

2013 "From Pictograph to Pictogram: The Solarization of Kingship in Syro-Anatolia and Assyria". Aruz et al. 2013, pp. 298-311.

POTTS, D. T.

2014 "Elamite Monumentality and Architectural Scale. Lessons from Susa and Choga Zanbil”. Osborne 2014, pp. 23-38.

RAINEY, A. F.

2015 The El-Amarna Correspondence. A New Edition of the Cuneiform Letters from the Site of El-Amarna based on Collations of all Extant Tablets. Handbook of Oriental studies. Section 1, The Near and Middle East 110. Leiden.

REDFORD, D. B

1976 "The Sun-disc in Akhenaten's Program: Its Worship and Antecedents I". Journal of the American Research Center in Egypt 13, pp. 47-61.

1980 "The Sun-disc in Akhenaten's Program: Its Worship and Antecedents II". Journal of the American Research Center in Egypt 17, pp. 21-38.

1984 Akhenaten, the Heretic King. Princeton.

2013 "Akhenaten: New Theories and Old Facts". Bulletin of the American Schools of Oriental Research 369, pp. 9-34. 
RLA. EBELING, E. ET AL.

1932ff. Reallexikon der Assyriologie und vorderasiatischen Archäologie. Berlin.

ROAF, M.

2017 «Kassite and Elamite Kings». Bartelmus/Sternitzke 2017, pp. 166-195.

SCHACHNER, A.

2011 Hattuscha. Auf der Suche nach dem sagenhaften Grossreich der Hethiter. München.

SCHMIDT, K. S. (HG.)

2015 Gedenkschrift für Mark A. Brandes (1929-2011). AOAT 423. Münster.

SCHWEMER, D.

2001 Die Wettergottgestalten Mesopotamiens und Nordsyriens im Zeitalter der Keilschriftkulturen. Materialien und Studien nach den schriftlichen Quellen. Wiesbaden.

SEIDL, U.

1989 Die babylonischen Kudurru-Reliefs. Symbole mesopotamischer Gottheiten. OBO

87. Fribourg.

SINGER, I.

2001 "The Fate of Hattusa during the Period of Tarhuntassa's Supremacy". Richter, T. ET AL. (Hgg.), 2001. Kulturgeschichten: altorientalistische Studien für Volkert Haas zum 65. Geburtstag. Saarbrücken, pp. 395-403.

STEIN, D.

1993 The Seal Impressions. Das Archiv des Šilwa-tě̌šup 8-9. Wiesbaden.

STEVENS, A.

2016 Tell el-Amarna. Wendrich, W. (ed.), UCLA Encyclopedia of Egyptology, Los Angeles. http://digital2.library.ucla.edu/viewItem.do?ark=21198/zz002k6x4x (7.6.2018).

STIEHLER-ALEGRIA DELGADO, G.

1996 Die Kassitische Glyptik. Münchener Vorderasiatische Studien 18. München.

TEISSIER, B.

1996 Egyptian Iconography on Syro-Palestinian Cylinder Seals of the Middle Bronze Age. Orbis Biblicus et Orientalis, Series Archaeologica 11. Fribourg.

TRAUNECKER, C.

1984 "Données nouvelles sur le début du règne d'Aménophis IV et son oeuvre à Karnak". Journal of the Society for the Study of Egyptian Antiquities 14.3, pp. 60-69.

TUAT NF 2. BREYER, F. ET AL. (HGG.)

2005 Staatsverträge, Herrscherinschriften und andere Dokumente zur politischen Geschichte. Texte aus der Umwelt des Alten Testaments. Neue Folge 2. Gütersloh. 
WÄFLER, M.

2003 Tall al-Hamīdīya 4. Vorbericht 1988-2001. Tall al-Hamīdīya 4. Orbis Biblicus et Orientalis. Series Archaeologica 23. Fribourg.

2007 «Tall al-Hamīdīya: Ta 'idu». Hefte des Archäologischen Seminars der Universität Bern 20, pp. 33-58.

WESTENDORF, W.,

1986 «Die Flügelsonne aus Ägypten». Archäologische Mitteilungen aus Iran 19, pp. $21-26$.

WILKINSON, R. H.

2000 The Complete Temples of Ancient Egypt. London.

YALCIN, S.

2011 "A Study of Cultural Interaction in the Eastern Mediterranean during Late Bronze Age: Adaptation of the Winged Sun Disc by the Hittites". Duistermaat/Regulski 2011, pp. 521-538. 
